\title{
Investigação de perdas devido ao trabalho inacabado e o seu impacto no tempo de ciclo dos processos construtivos
}

\author{
Investigation of wastes due to unfinished work and their \\ impact on the cycle time of construction processes
}

\section{Paulo Ricardo Ramos Santos Débora de Gois Santos}

\section{Resumo \\ A} $\mathrm{s}$ perdas na construção civil constituem um entrave à melhoria da produtividade. Dentre os diversos tipos de perdas, o making-do se destaca por representar uma fonte de outras perdas. Fireman et al. (2013), ao estudarem o making-do, concluíram que ele pode ser desencadeado por um tipo de perda geral, denominada de trabalho inacabado. $\mathrm{O}$ objetivo do presente trabalho foi investigar a ocorrência do trabalho inacabado nos processos construtivos e o seu impacto no tempo de ciclo dos serviços. A pesquisa foi de um levantamento de campo em cinco canteiros, envolvendo observação direta. Utilizou-se como ferramentas de pesquisa: formulário, questionários e checklist, adaptados da literatura para identificação e classificação do trabalho inacabado, bem como aferição do seu impacto no tempo de ciclo. Houve também entrevistas não estruturadas com os gerentes de obras, mestres e encarregados. Os resultados mostraram que as falhas no sequenciamento das tarefas associadas com falhas na gestão da qualidade serviram de desencadeadores para o trabalho inacabado. Notou-se que os canteiros que apresentaram planos de curto prazo formalizados atenuaram os impactos desse tipo de perda. Na maioria das obras o tempo de ciclo previsto não foi cumprido, observando-se que aquelas que tinham equipe administrativa mais encorpada obtiveram acréscimo de tempo menor.

Palavras-chaves: Construção enxuta. Making-do. Trabalho inacabado.
Paulo Ricardo Ramos Santos Universidade Federal de Sergipe Sáo Cristovão - SE - Brasil

Débora de Gois Santos Universidade Federal de Sergipe Sáo Cristovão - SE - Brasil

Recebido em 04/03/16 Aceito em 18/08/16

\begin{abstract}
Construction waste constitutes an obstacle for the improvement of productivity. Among the different kinds of construction waste, making-do stands out as a source of other types of waste. When Fireman et al. (2013) studied making-do waste, they concluded that it could be triggered by a kind of general waste called unfinished work. The aim of this study was to investigate the occurrence of unfinished work in construction processes and its impact on the cycle time of services. The study consisted in a field survey carried out on five construction sites, involving direct observation. The research tools used were forms, questionnaires and checklists, all adapted from the literature, with the aim of identifying and classifying unfinished work. In addition, semi-structured interviews were conducted with site managers and supervisors. Time measurements were taken to check the impact of unfinished work on cycle time. The results showed that failures in the sequence of tasks associated with failures in quality management were triggers for the unfinished work. The study showed that the construction sites that had formalised short-term plans were able to mitigate the impacts of this type of waste. On most of the construction sites investigated, the planned cycle time was not met, and also that those sites that had larger management teams presented lower added time.

Keywords: Lean construction. Making-do. Unfinished work.
\end{abstract}




\section{Introdução}

O mercado da construção civil está cada vez mais competitivo. O período de crescimento do início da década de 2010 passou e deu lugar a um ambiente incerto, com pouca previsão de rentabilidade para as construtoras. Esse cenário exige um investimento maior no planejamento $\mathrm{e}$ gerenciamento das obras. Desse modo, as empresas que obtiverem um controle mais eficiente da produção e da qualidade serão mais competitivas.

Vários estudos de diferentes países confirmaram que as perdas representam uma porcentagem relativamente grande dos custos de produção (VIANA; FORMOSO; KALSAAS, 2012). A construção enxuta propõe a redução de perdas e improvisos na produção em busca de um produto de maior qualidade e menos custo. Sendo assim, a eliminação de perdas tem sido a palavra-chave para as empresas que adotaram essa filosofia gerencial. (SOMMER, 2010; BARBOSA et al., 2013).

Dentre os diversos tipos de perdas, o making-do se destaca por representar uma fonte de outras perdas como redução da segurança, problemas de qualidade, work in progress e retrabalho (SOMMER, 2010; FORMOSO et al., 2011; FIREMAN et al., 2013). Fireman et al. (2013), ao estudarem o making-do mais a fundo, concluíram que ele pode ser desencadeado por um tipo de perda geral, que os autores denominaram de trabalho inacabado.

Questiona-se, então, qual o impacto do trabalho inacabado no tempo de ciclo de processos construtivos?

O objetivo desta pesquisa foi investigar a ocorrência do trabalho inacabado nos processos construtivos, analisando a influência do controle da produção para sua ocorrência e o seu impacto no tempo de ciclo dos serviços.

\section{Making-do e trabalho inacabado}

O termo making-do foi idealizado por Koskela (2004) para definir a perda que ocorre quando uma tarefa inicia sem todas as suas entradas, ou quando ela tem sua execução continuada, mesmo com a cessão de uma ou mais entradas. Para Sommer (2010), making-do significa improvisação, no sentido de executar uma atividade com o que se tem disponível ou na linguagem mais coloquial "dar um jeitinho".
De acordo com Weick (1998) apud Sommer (2010), a improvisação está relacionada a como as pessoas lidam com a falta de recursos para concluir suas atividades sem obter prejuízos. Cunha (2004) trata a improvisação como um sinônimo de bricolagem, significando a invenção de recursos, a partir do que está disponível, com o intuito de resolver problemas antecipadamente.

Koskela (2004) observa que o modelo de gestão e planejamento da construção está todo voltado para a tarefa iniciada, ao passo que as discrepâncias entre o que foi planejado e o mundo real não são levadas em conta, o que resulta em situações nas quais o making-do aparece como única solução. Em suma, o referido autor conclui que o oitavo tipo de perda - como é conhecido o making-do não é fruto de uma falha da implantação do sistema de gestão convencional, mas sim uma causa dele.

O estudo de perdas decorrentes do making-do está bem difundido por diversos pesquisadores (SOMMER, 2010; FORMOSO et al., 2011; FIREMAN et al., 2013; LEÃO, 2014). Entretanto, anteriormente autores como Machado (2003) e Santos (2004) destacaram a importância da análise de restrições para planejar e antecipar os recursos necessários para a execução e continuidade da tarefa, participando como fontes de entendimento de conceitos essenciais e predecessores do surgimento do making-do.

Machado (2003) estudou o uso sistemático de antecipações gerenciais no planejamento da produção. Para esse autor, essas antecipações seriam fruto da experiência de projetos anteriores, visando o provimento dos recursos necessários para a execução dos serviços. No seu estudo, o autor chamou de antecipação as ações de proteção da produção tomadas pelo gerente para garantir a realização de todas as operações antecedentes do processamento. Ele também propôs categorias para as antecipações gerenciais.

Santos (2004) propôs um conceito de atividade que, por meio de remoção de restrição ou por antecipação gerencial, traz melhorias a um determinado processo. As atividades que se enquadram nesse conceito também podem ser entendidas como atividades de boas práticas. As boas práticas são ações de melhorias implementadas em um determinado setor, para dar mais eficiência e eficácia aos processos (TREVILLE; ANTONAKIS, 2006).

Quando as boas práticas contribuem para evitar interrupções no processo são chamadas de atividades facilitadoras (SANTOS, 2004). Elas se

40 Santos, P. R. R.; Santos, D. de G. 
relacionam com o conceito de melhoria contínua (KOSKELA, 1992), favorecendo um fluxo contínuo de produção e envolvendo a incorporação de informações ao processo que evitarão mais tarde nas condições subótimas de trabalho (KOSKELA, 2000). Elas também minimizam eventuais problemas de entradas que viriam a desfigurar o kit completo (RONEN, 1992).

Santos (2004, p. 91) assim denominou atividades facilitadoras:

As atividades facilitadoras são definidas
como ações que quando inseridas no
sistema de produção impedem ou
minimizam as paradas, ou seja, as
descontinuidades ao longo do processo
produtivo. Elas podem estar incorporadas
no processo ou auxiliá-lo. Apresentam-se
como atividades de produção, de apoio à
produção ou ainda como informações.

Tais atividades foram ainda divididas em nove categorias pela autora: acesso; projeto; preparação do trabalho; conferência do trabalho; conflito espacial; sequenciamento; proteção dos operários; proteção dos processos; e programação de obra.

Sommer (2010) propôs um método de identificação do making-do nos canteiros de obras
(Figura 1). A mesma autora apoiou-se nos sete fluxos propostos por Koskela (2000) para criar categorias de precondições que ao faltarem causam o making-do. A autora também criou sete categorias para classificação do making-do. No Quadro 1 essas categorias são listadas, bem como, é listada também a categoria "Sequenciamento", proposta por Fireman (2012) e aplicada por Leão (2014).

Machado (2003) e Santos (2004) propuseram uma lista de ações gerenciais para evitar a interrupção e Sommer (2010) apresentou alternativas encontradas pelos operários para as interrupções, criando as categorias de perdas por improvisações.

De acordo com Sommer (2010), a interação entre quem planeja e quem executa é fundamental para evitar as perdas decorrentes do making-do, sobretudo quanto a uma análise prévia dos requisitos necessários para a execução dos pacotes de trabalho.

Fireman (2012) identificou que muitos pacotes existiam pela falta de terminalidade de pacotes anteriores. Tais pacotes ocorriam por falta de arremate ou por falta do total processamento de alguns elementos construtivos.

\section{Figura 1 - Método de identificação de perdas por making-do (improvisação)}

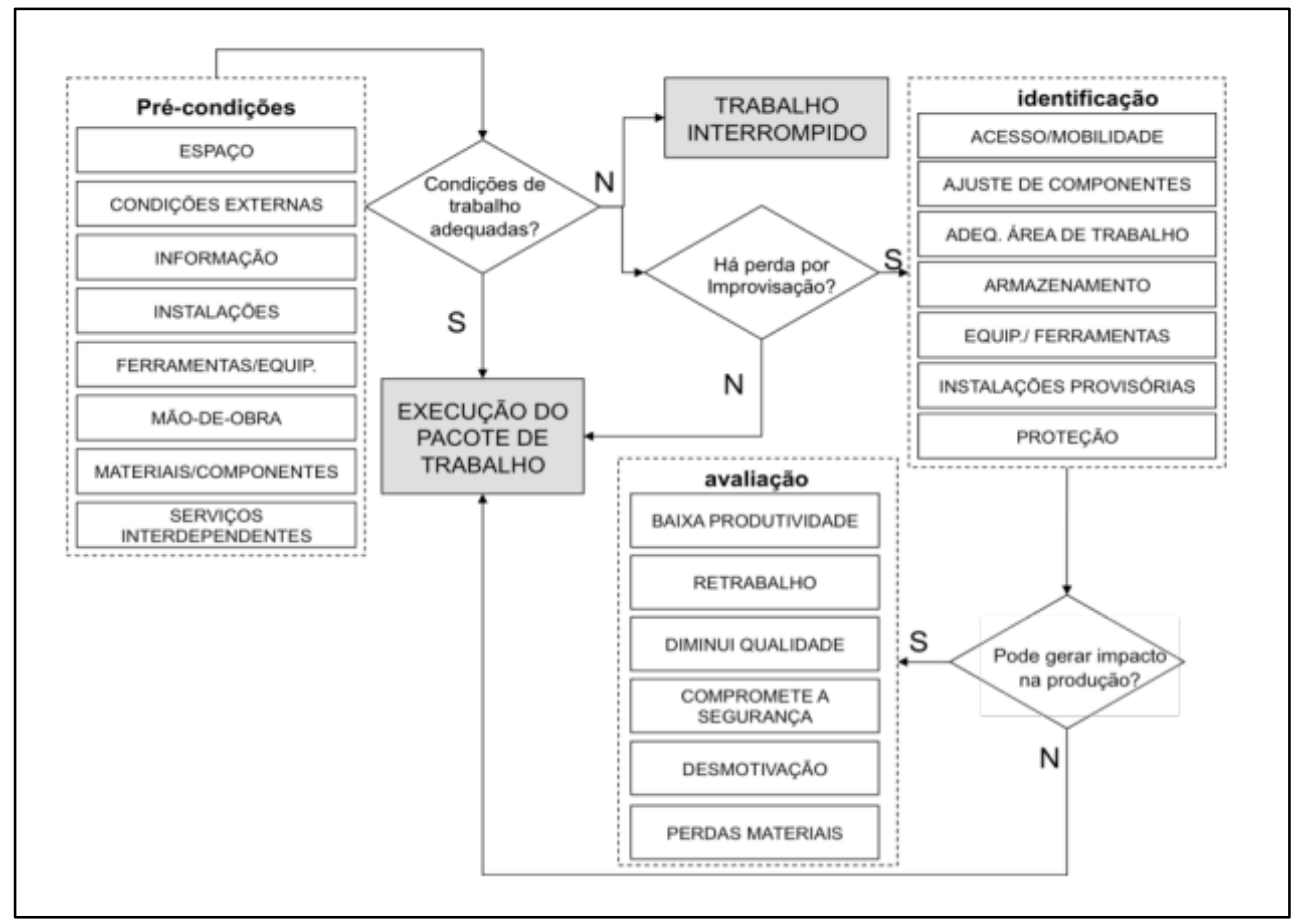

Fonte: Sommer (2010). 
Quadro 1 - Classificação de perda por making-do

\begin{tabular}{|c|c|c|c|c|c|}
\hline $\begin{array}{l}\text { IDENTIFICAÇÃO/ } \\
\text { CATEGORIA }\end{array}$ & AUTOR & $\begin{array}{c}\text { PRÉCONDIÇÃO/ } \\
\text { PRÉ-REQUISITOS } \\
\text { QUE FALTAM }\end{array}$ & AUTOR & $\begin{array}{c}\text { IMPACTO/ } \\
\text { AVALIAÇÃO }\end{array}$ & AUTOR \\
\hline Acesso/mobilidade & Sommer (2010) & Informação & $\begin{array}{l}\text { Sommer (2010); } \\
\text { Koskela (2000) }\end{array}$ & $\begin{array}{c}\text { Baixa } \\
\text { produtividade }\end{array}$ & $\begin{array}{l}\text { Sommer } \\
(2010)\end{array}$ \\
\hline $\begin{array}{c}\text { Ajustes de } \\
\text { componentes }\end{array}$ & Sommer (2010) & $\begin{array}{c}\text { Materiais e } \\
\text { componentes }\end{array}$ & $\begin{array}{l}\text { Sommer (2010); } \\
\text { Koskela (2000) }\end{array}$ & $\begin{array}{c}\text { Diminuição da } \\
\text { qualidade }\end{array}$ & $\begin{array}{l}\text { Sommer } \\
(2010)\end{array}$ \\
\hline Área de trabalho & Sommer (2010) & Mão de obra & $\begin{array}{l}\text { Sommer (2010); } \\
\text { Koskela (2000) }\end{array}$ & Retrabalho & $\begin{array}{l}\text { Sommer } \\
(2010)\end{array}$ \\
\hline $\begin{array}{l}\text { Armazenamento: } \\
\text { estoque de } \\
\text { materiais ou } \\
\text { componentes } \\
\end{array}$ & Sommer (2010) & $\begin{array}{c}\text { Equipamentos/ferra- } \\
\text { mentas }\end{array}$ & $\begin{array}{l}\text { Sommer (2010); } \\
\text { Koskela (2000) }\end{array}$ & $\begin{array}{l}\text { Perdas de } \\
\text { materiais }\end{array}$ & $\begin{array}{l}\text { Sommer } \\
(2010)\end{array}$ \\
\hline $\begin{array}{c}\text { Equipamentos/ } \\
\text { ferramentas }\end{array}$ & Sommer (2010) & Espaço & $\begin{array}{l}\text { Sommer (2010); } \\
\text { Koskela (2000) } \\
\end{array}$ & $\begin{array}{c}\text { Compromete a } \\
\text { segurança }\end{array}$ & $\begin{array}{l}\text { Sommer } \\
(2010)\end{array}$ \\
\hline \begin{tabular}{|c|} 
Instalação \\
provisória: \\
suprimento de água \\
e eletricidade \\
\end{tabular} & Sommer (2010) & Serviços interligados & $\begin{array}{l}\text { Sommer (2010); } \\
\text { Koskela (2000) }\end{array}$ & Desmotivação & $\begin{array}{l}\text { Sommer } \\
(2010)\end{array}$ \\
\hline Proteção & Sommer (2010) & Condições externas & $\begin{array}{l}\text { Sommer (2010); } \\
\text { Koskela (2000) }\end{array}$ & $\begin{array}{c}\text { Falta de } \\
\text { terminalidade } \\
\end{array}$ & $\begin{array}{l}\text { Fireman } \\
(2012)\end{array}$ \\
\hline Sequenciamento & Fireman (2012) & $\begin{array}{c}\text { Instalações: } \\
\text { infraestrutura do } \\
\text { espaço de trabalho }\end{array}$ & Sommer (2010) & & \\
\hline
\end{tabular}

Fireman et al. (2013) exploraram uma categoria de perda adicional, denominada trabalho inacabado. O trabalho inacabado consiste em pacotes de trabalho que não são executados dentro do seu prazo previsto e acabam por gerar outros pequenos pacotes, que muitas vezes são desprezados na elaboração dos próximos planos de curto prazo, o que implica acréscimo no tempo de ciclo e perdas de mão de obra (FIREMAN et al., 2013).

Fireman (2012) tratou os pacotes de curto prazo, que não são planejados, como informais e os separou em três categorias:

(a) falta de terminalidade - representa que uma tarefa considerada concluída na semana anterior podia ainda estar em execução durante as semanas seguintes. Essa tarefa poderia estar associada a alguns elementos (ex.: uma parede inteira) que faltavam ser executados ou mesmo pequenos detalhes (arremates);

(b) novos - refere-se à antecipação de uma tarefa que, de acordo com a sequência do ciclo, iria ser iniciada apenas na semana seguinte; e

(c) retrabalho - atividades relacionadas à correção de pacotes executados sem qualidade, no ciclo de produção anterior.

Os impactos/avaliações "falta de terminalidade" e "retrabalho" são provenientes de processos inconclusos ou concluídos com má qualidade e constituem o trabalho inacabado na produção.

Um mapa conceitual com base nos trabalhos de Machado (2003), Santos (2004), Sommer (2010), principalmente, e Fireman (2012) pode ser utilizado para ilustrar as inter-relações entre os conceitos: making-do, atividades facilitadoras e trabalho inacabado, bem como suas categorias/classificações (Figura 2).

O impacto do trabalho inacabado no tempo de ciclo (lead time) dos processos é inevitável, uma vez que o trabalho é interrompido e são necessárias novas operações de mobilização e desmobilização, além de novo processamento. Com isso, o tempo de ciclo aumenta e o pacote de trabalho, que agora é informal, oferece um ambiente ideal para a ocorrência de making-do (ALVES, 2000; FORMOSO et al., 2011; FIREMAN et al., 2013).

Embora Fireman (2012) ainda não tivesse usado o termo trabalho inacabado, alguns exemplos de pacotes informais citados em seu trabalho se encaixam nesse tipo de perda (Figuras 3 e 4). A Figura 3 mostra rasgos na alvenaria para correção de pontos elétricos - retrabalho. Já a Figura 4 mostra paredes de um shaft que não puderam ser concluídas porque as prumadas de esgoto não estavam completas - falta de terminalidade. 
Figura 2 - Mapa conceitual com inter-relações entre os conceitos que embasam a pesquisa

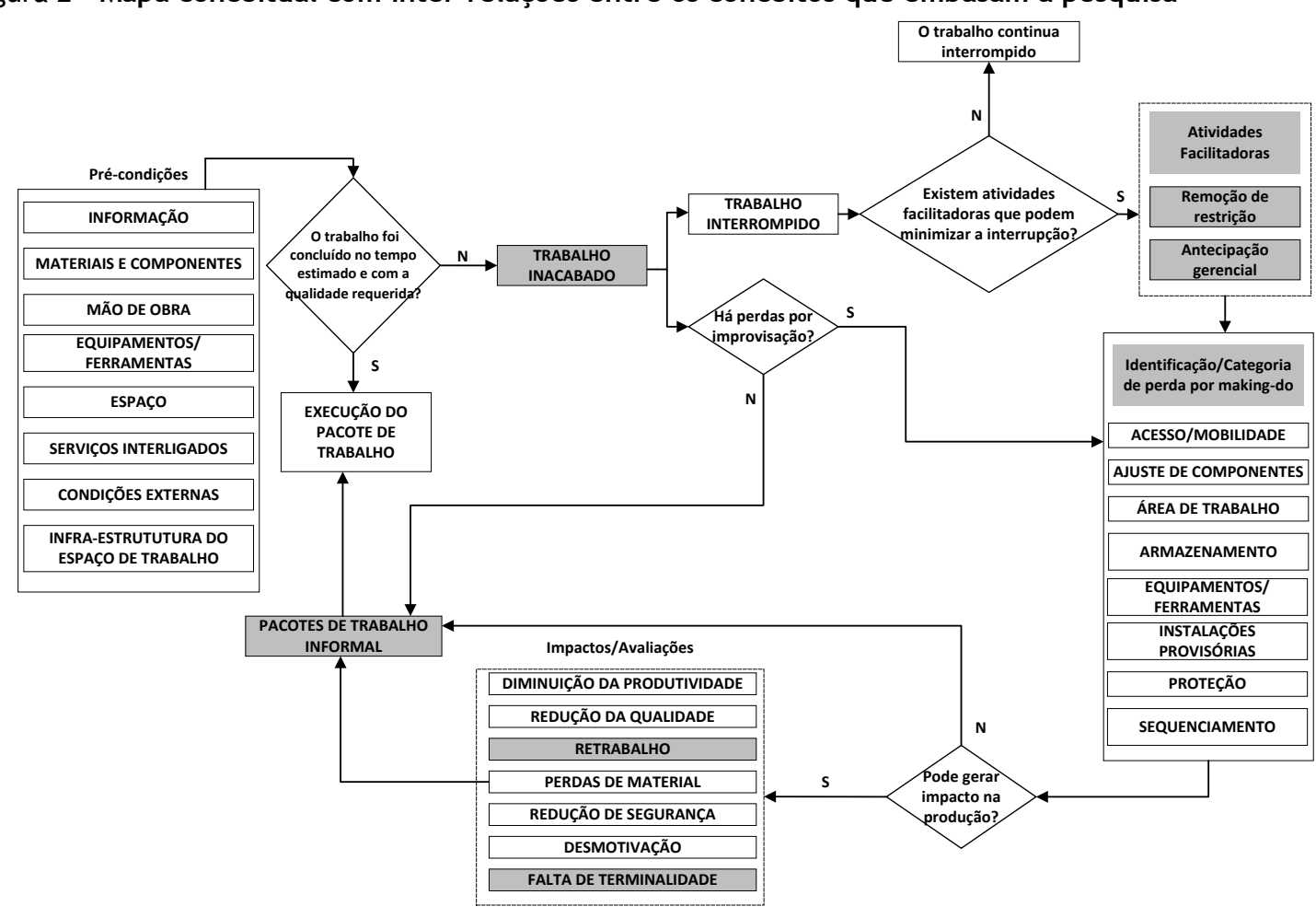

Figura 3 - Exemplo de trabalho inacabado em alvenaria - retrabalho

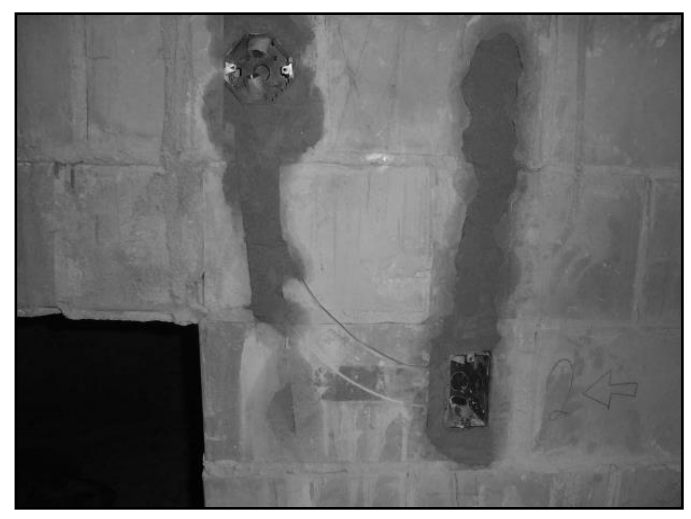

Fonte: Fireman (2012)

Figura 4 - Exemplo de trabalho inacabado - falta de terminalidade

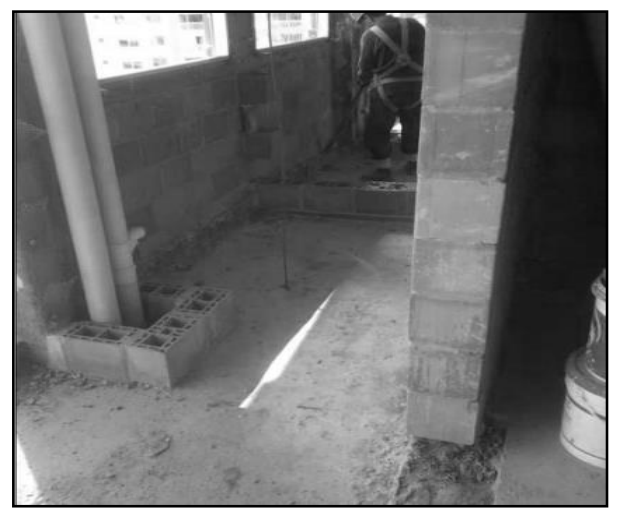

Fonte: Fireman (2012). 


\section{Método}

O delineamento da pesquisa foi de levantamento de campo em canteiros de obra, que ocorreu por intermédio de estudo exploratório e descritivo, com pesquisa qualitativa. As ferramentas de pesquisa utilizadas foram: formulário para identificação do trabalho inacabado, questionários para classificação do trabalho inacabado (adaptados da literatura), checklist de planejamento e controle da produção (PCP), registro fotográfico, consulta a projetos e documentos, além de entrevistas não estruturadas com os gerentes de obras e encarregados.

Os cinco objetos de pesquisa foram empreendimentos que estavam executando serviços internos de acabamento, entre eles, revestimento cerâmico interno (Quadro 2). A escolha desse serviço ocorreu pelo tempo de ciclo curto que ele apresenta e por envolver atividades do final do processo construtivo, em que os efeitos de falhas são mais visíveis.

Os dados foram obtidos a partir de observação direta, pelo acompanhamento de ciclos do serviço escolhido, que se encontrava em vários estágios de execução durante as visitas. Em cada obra ocorreu uma visita em um dia de trabalho. Quando havia indicação de trabalho inacabado o mestre ou os encarregados das equipes envolvidas eram questionados sobre os motivos e um dos pesquisadores preenchia $\mathrm{o}$ formulário para identificação do trabalho inacabado.

Após a observação em campo eram aplicados aos profissionais citados os questionários sobre as identificações/categorias, os pré-requisitos e os impactos/avaliação do tipo de perda investigada, a fim de obter sua classificação.

Os critérios de classificação do making-do, encontrados em outras pesquisas, foram utilizados como referência para classificar o trabalho inacabado (FORMOSO et al., 2011; FIREMAN et al., 2013; LEÃO, 2014).

O formulário para identificação (Figura 5) foi construído a partir do formulário de identificação de perdas por improvisação de Sommer (2010), com questões adaptadas dos trabalhos de Formoso et al. (2011), Fireman (2012) e Fireman et al. (2013). Os questionários de classificação também tiveram como base os mesmos trabalhos, sendo constituídos de questões norteadoras.

\section{Quadro 2 - Características dos estudos de caso}

\begin{tabular}{|c|c|c|c|c|c|}
\hline \multirow{2}{*}{$\begin{array}{c}\text { OBRA } \\
\text { A }\end{array}$} & \multicolumn{2}{|c|}{$\begin{array}{l}\text { CARACTERÍSTICAS DO } \\
\text { EMPREENDIMENTO }\end{array}$} & \multicolumn{2}{|c|}{ EQUIPE DE ENGENHARIA } & \multirow{2}{*}{$\begin{array}{c}\text { EQUIPE DE } \\
\text { REVESTIMENTO } \\
\text { CERÂMICO } \\
\\
8 \text { oficiais } \\
4 \text { serventes }\end{array}$} \\
\hline & 1 torre & $\begin{array}{c}13 \text { Pav. Tipos } \\
1 \text { Playground } \\
1 \text { Pav. Garagem } \\
\text { Área privativa apto padrão: } \\
117,22 \mathrm{~m}^{2}\end{array}$ & $\begin{array}{l}1 \text { engenheiro } \\
1 \text { assistente de } \\
\text { engenharia }\end{array}$ & $\begin{array}{l}1 \text { mestre de obras } \\
3 \text { estagiários }\end{array}$ & \\
\hline B & 2 torres & $\begin{array}{c}\text { 13 Pav. Tipos } \\
\text { 1 Playground } \\
\text { 2 Pav. Garagem } \\
\text { Área privativa apto padrão: } \\
153,62 \mathrm{~m}^{2} \\
\end{array}$ & $\begin{array}{l}3 \text { engenheiros } \\
2 \text { assistentes de } \\
\text { engenharia }\end{array}$ & $\begin{array}{c}1 \text { mestre de obras } \\
3 \text { estagiários }\end{array}$ & $\begin{array}{l}12 \text { oficiais } \\
5 \text { serventes }\end{array}$ \\
\hline C & 4 torres & $\begin{array}{c}14 \text { Pav. Tipos } \\
1 \text { Pav. Garagem } \\
\text { Área privativa apto padrão: } \\
59 \mathrm{~m}^{2}\end{array}$ & $\begin{array}{l}4 \text { engenheiros } \\
4 \text { assistentes de } \\
\text { engenharia }\end{array}$ & $\begin{array}{l}5 \text { mestre de obras } \\
2 \text { técnicos em } \\
\text { edificações }\end{array}$ & $\begin{array}{l}42 \text { oficiais } \\
21 \text { serventes }\end{array}$ \\
\hline D & 2 torres & $\begin{array}{c}\text { 13 Pav. Tipos } \\
\text { 1 Playground } \\
\text { 1 Pav. Garagem } \\
\text { Área privativa apto padrão: } \\
61 \mathrm{~m}^{2} \\
\end{array}$ & 2 engenheiros & $\begin{array}{l}1 \text { mestre de obras } \\
2 \text { técnicos em } \\
\text { edificações } \\
2 \text { estagiários } \\
\end{array}$ & $\begin{array}{l}21 \text { oficiais } \\
10 \text { serventes }\end{array}$ \\
\hline $\mathbf{E}$ & 4 torres & $\begin{array}{c}\text { 12 Pav. Tipos } \\
\text { 1 Playground } \\
\text { 2 Pav. Garagem } \\
\text { Área privativa apto padrão: } \\
101,77 \mathrm{~m}^{2}\end{array}$ & $\begin{array}{l}2 \text { engenheiros } \\
1 \text { assistente de } \\
\text { engenharia }\end{array}$ & $\begin{array}{l}1 \text { mestre de obras } \\
1 \text { contramestre } \\
4 \text { estagiários }\end{array}$ & $\begin{array}{l}20 \text { oficiais } \\
8 \text { serventes }\end{array}$ \\
\hline
\end{tabular}

44 Santos, P. R. R.; Santos, D. de G. 
Figura 5 - Formulário de identificação do trabalho inacabado

\begin{tabular}{|c|c|}
\hline \multirow{2}{*}{ Qual a causa do pacote informal? } & Falta de terminalidade \\
\hline & Retrabalho \\
\hline \multirow{2}{*}{ Qual o motivo da não conclusão? } & Tarefa anterior \\
\hline & Falta de entradas padrão \\
\hline \multirow{2}{*}{ O trabalho foi finalizado com improvisação (making-do)? } & Sim \\
\hline & Não \\
\hline
\end{tabular}

A primeira questão tratou do motivo da não conclusão do serviço, se foi por conta da tarefa anterior, que deixou de ser executada ou foi mal feita, ou por conta de falta de entradas padrão. A segunda questão tratou de identificar o tipo de pacote informal gerado pelo trabalho inacabado, de acordo com os pacotes informais propostos por Fireman (2012). Considerou-se que a ocorrência do trabalho inacabado pode vir ou não acompanhada de making-do (terceira questão).

A base de classificação inicial foi a de Sommer (2010). A autora propôs um método para identificação de perdas por improvisação que tratou das precondições, identificação e avaliação dessas perdas. As precondições propostas pela autora foram baseadas nos setes fluxos de entrada propostos por Koskela (2000). Posteriormente, Fireman (2012) acrescentou uma categoria de precondição e outra de impacto, que foram aqui utilizadas. O Quadro 1 apresenta a categorização para cada uma das classificações.

O checklist de PCP (Figura 6) foi elaborado pelos autores como uma forma de identificar a relação do PCP da empresa com o trabalho inacabado. As perguntas do checklist foram respondidas pelos engenheiros responsáveis pelos planejamentos dos canteiros, mestres e encarregados do serviço de cerâmica nas obras.

Para investigação do impacto do trabalho inacabado no tempo de ciclo, utilizaram-se dois indicadores a partir de dados informados pela equipe da obra (indicadores A e B) (Figura 7). A unidade adotada para coleta em campo foi dias de trabalho, por ser mais utilizada no cotidiano das obras. Para discussão dos dados, utilizou-se a unidade homem-hora de trabalho (Hh), multiplicando-se cada dia por $8,8 \mathrm{~h}$ vezes o número de oficiais da equipe.

$\mathrm{O}$ indicador (A) foi o indicador formal e referiu-se ao tempo estimado pelo engenheiro para a execução em campo, de acordo com o planejamento do serviço. Já o indicador (B) foi o indicador real que estava sendo obtido, de acordo com o tempo médio de execução, também informado pelo engenheiro de cada obra.

$\mathrm{O}$ indicador (C) foi calculado e apresentou a quantidade de dias gasta na realização do pacote informal, considerada a subtração de (B) - (A). O indicador (D) representou a percentagem de pacotes informais, obtido pela divisão de (C) sobre (B) vezes 100 .

Considerou-se que todo o tempo extra para a realização do trabalho foi despendido na forma de pacote informal, baseando-se na hipótese de que o pacote informal toma a maior parte desse tempo por envolver diversos tipos de perda, inclusive o making-do. Além disso, considerou-se que ele incorpora todo o tempo decorrente de novas mobilizações e de outras atividades que não agregam valor, advindas da não conclusão dos serviços e do aumento do work in progress (FIREMAN, 2012; FIREMAN et al., 2013).

\section{Resultados e discussão}

Quanto ao formulário para a identificação do trabalho inacabado, obtiveram-se como resumo das obras os dados apresentados na Figura 8.

Exemplos de trabalho finalizado com making-do foram encontrados em duas das três obras pesquisadas (Figura 8) e foram frutos da improvisação dos trabalhadores que ajustaram componentes construtivos para concluir o seu trabalho. Nas mesmas obras onde se encontraram os exemplos de making-do (obras A e C) existiram também pacotes informais de retrabalho, o que mostra que a improvisação levou à perda de qualidade do serviço. 
Figura 6 - Checklist de planejamento e controle da produção

\begin{tabular}{|c|c|c|c|c|c|c|c|c|c|c|}
\hline \multirow{2}{*}{ ENGENHEIRO } & \multicolumn{2}{|c|}{ Obra A } & \multicolumn{2}{|c|}{ Obra B } & \multicolumn{2}{|c|}{ Obra C } & \multicolumn{2}{|c|}{ Obra D } & \multicolumn{2}{|c|}{ Obra E } \\
\hline & $\operatorname{Sim}$ & Não & $\operatorname{Sim}$ & Não & $\operatorname{Sim}$ & Não & $\operatorname{Sim}$ & Não & $\operatorname{Sim}$ & Não \\
\hline \multicolumn{11}{|l|}{ Os serviços semanais são plane jados formalmente? } \\
\hline \multicolumn{11}{|l|}{ Há reunião conjunta entre empreiteira e terceirizadas? } \\
\hline \multicolumn{11}{|l|}{ São levantadas possíveis restrições nos plane jamentos? } \\
\hline \multicolumn{11}{|l|}{ Existe preocupação em observar as condições de campo para os seviços futuros? } \\
\hline \multicolumn{11}{|l|}{ Quando ocorre atraso existe há inves tigação do motivo? } \\
\hline \multirow{2}{*}{ ENCARREGADO OU MESTRE } & \multicolumn{2}{|c|}{ Obra A } & \multicolumn{2}{|c|}{ Obra B } & \multicolumn{2}{|c|}{ Obra C } & \multicolumn{2}{|c|}{ Obra D } & \multicolumn{2}{|c|}{ Obra E } \\
\hline & Sim & Não & Sim & Não & Sim & Não & Sim & Não & Sim & Não \\
\hline \multicolumn{11}{|l|}{ As equipes iniciais são as que fazem o retrabalho? } \\
\hline \multicolumn{11}{|l|}{ Geralmente o ope rário realiza serviços parecidos? } \\
\hline \multicolumn{11}{|l|}{ O serviço não foi interrompido por modificação de projeto? } \\
\hline \multicolumn{11}{|l|}{ Existe FVS para os serviços executados? } \\
\hline \multicolumn{11}{|l|}{ Os motivos das não conclusões são registrados? } \\
\hline \multicolumn{11}{|l|}{ As FVS's são analisadas para os novos planejamentos? } \\
\hline \multicolumn{11}{|l|}{ Existem ações corretivas com base nas restrições identificadas? } \\
\hline A qualidade do serviço é ins pecionada durante a execução? & & & & & & & & & & \\
\hline As frentes de trabalho só são liberadas com a aprovação da qualidade das anteriores? & & & & & & & & & & \\
\hline
\end{tabular}

Figura 7 - Indicadores de tempo para detectar o impacto do trabalho inacabado no tempo de ciclo

\begin{tabular}{|c|c|c|c|}
\hline \multirow{2}{*}{ (A) } & Tempo de realização previsto pela programação & Dias & Hh \\
\cline { 3 - 4 } & & (Formal) & \\
\hline (B) & $\begin{array}{c}\text { Tempo de realização do serviço } \\
\text { (Real) }\end{array}$ & Dias & Hh \\
\cline { 3 - 4 } & Hh gasto na realização do pacote informal & & \\
\hline (C) = (B - A) & Percentual de tempo de pacotes informais & \\
\hline (D) $=$ (C/B)*100 & & & \\
\hline
\end{tabular}

Figura 8 - Formulário do trabalho inacabado nas obras

\begin{tabular}{|c|c|c|c|c|c|c|c|}
\hline \multirow{2}{*}{ Qual a causa do pacote informal? } & Obra A & Obra B & Obra C & Obra D & Obra E & Total \\
\hline & Falta de terminalidade & $\mathrm{X}$ & $\mathrm{X}$ & $\mathrm{X}$ & $\mathrm{X}$ & $\mathrm{X}$ & 5 \\
\hline \multirow{2}{*}{ Qual o motivo da não conclusão? } & Retrabalho & $\mathrm{X}$ & & $\mathrm{X}$ & & $\mathrm{X}$ & 3 \\
\hline & Tarefa anterior & $\mathrm{X}$ & & $\mathrm{X}$ & $\mathrm{X}$ & $\mathrm{X}$ & 4 \\
\hline $\begin{array}{c}\text { O trabalho foi finalizado com } \\
\text { improvisação (making-do )? }\end{array}$ & $\mathrm{F}$ Sim & $\mathrm{X}$ & & $\mathrm{X}$ & & & 2 \\
\cline { 2 - 8 } & $\mathrm{Não}$ & & $\mathrm{X}$ & & $\mathrm{X}$ & $\mathrm{X}$ & 3 \\
\hline
\end{tabular}

Na Figura 9 nota-se um exemplo de making-do. O pedreiro, para concluir o seu serviço, mesmo com o erro do processo anterior, resolveu improvisar e adaptar a placa cerâmica à tubulação do arcondicionado mal posicionada. Outros exemplos desse tipo foram encontrados nas obras.

Em todas as obras encontraram-se pacotes informais devido à falta de terminalidade, característicos do trabalho inacabado (Figura 8). Em três delas identificou-se também pacotes de retrabalho. Nessa situação, o retrabalho ocorreu devido à má qualidade dos elementos produzidos, o que também interferiu na não conclusão do trabalho.

Na Figura 10 tem-se um exemplo de retrabalho devido à falta de qualidade no acabamento. No caso, o tamanho do recorte na placa cerâmica era maior do que a peça de acabamento do registro, o que deixou rebarbas aparentes.

A má qualidade nos casos de retrabalho estava também associada à qualidade dos produtos/processos anteriores, que apresentavam 
inconformidades provenientes das falhas da gestão da qualidade. Aqui, vale ressaltar a influência do PCP para a ocorrência do trabalho inacabado. As duas obras que não apresentaram pacotes de retrabalho (obras B e D) tinham um controle rígido na correção dos processos (serviços de construção) para a liberação de frente de trabalho. Nos dois casos também foi comum a discussão de possíveis restrições para o trabalho e a ação a partir da prevenção por parte dos gestores (Figura 11).

Percebeu-se que falhas no sequenciamento das atividades contribuíram para o trabalho inacabado nessas obras. A defasagem entre a equipe de revestimento cerâmico e as equipes de serviços anteriores prejudicou a terminalidade do serviço e criou gargalos para a produção. Algumas obras contornaram esse problema com a realocação das equipes de revestimento cerâmico enquanto o serviço anterior pendente era concluído, o que evitou a interrupção do trabalho dos operários.

A única obra a não apresentar trabalho inacabado por tarefas anteriores foi a obra B. Porém, essa obra apresentou falta de terminalidade devido à falta de insumos necessários para o serviço. Falta essa proveniente da customização dos apartamentos e de falha logística na entrega de materiais por parte dos clientes (Figura 12).

A classificação geral em categorias do trabalho inacabado e os pré-requisitos relacionados à sua existência podem ser visualizados nas Figuras $13 \mathrm{e}$ 14.

\section{Figura 9 - Tubulação do ar condicionado impediu o correto assentamento da placa cerâmica}

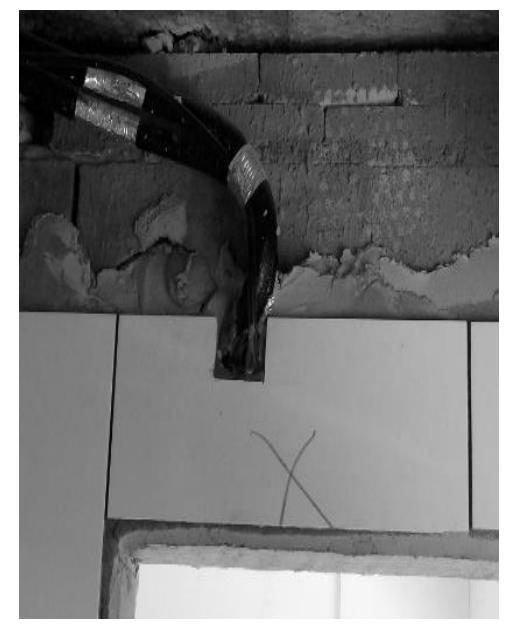

Figura 10 - Rebarba no ponto de água fria

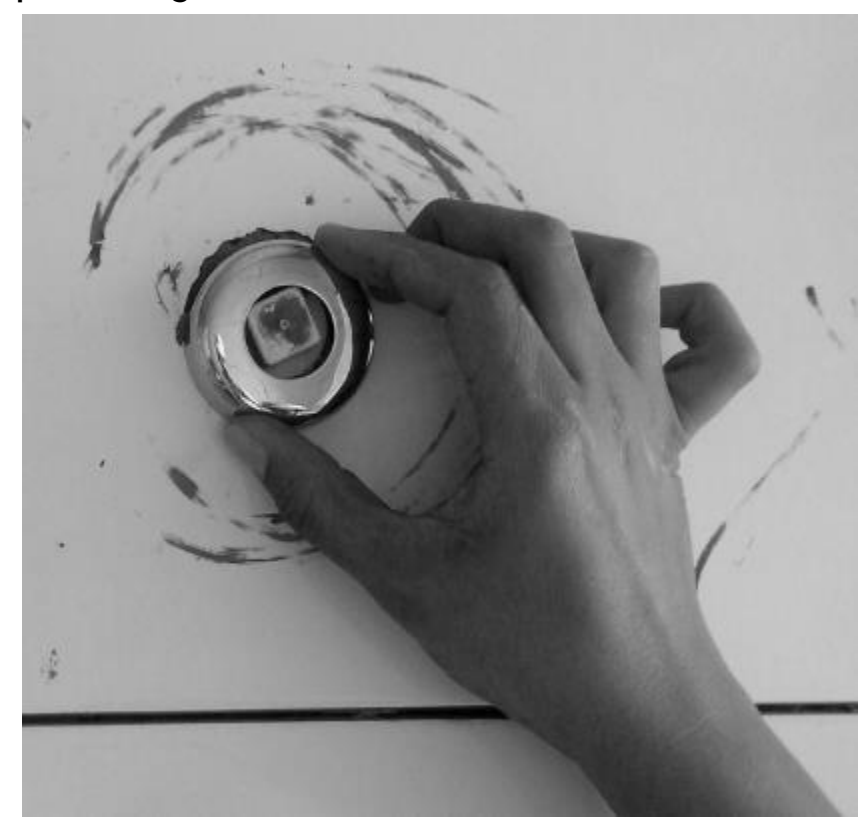


Figura 11 - Resultado da aplicação do checklist de planejamento e controle de produção

\begin{tabular}{|c|c|c|c|c|c|c|c|c|c|c|}
\hline \multirow{2}{*}{ ENGENHEIRO } & \multicolumn{2}{|c|}{ Obra A } & \multicolumn{2}{|c|}{ Obra B } & \multicolumn{2}{|c|}{ Obra $\mathrm{C}$} & \multicolumn{2}{|c|}{ Obra D } & \multicolumn{2}{|c|}{ Obra E } \\
\hline & $\operatorname{Sim}$ & Não & $\operatorname{Sim}$ & Não & $\operatorname{Sim}$ & Não & Sim & Não & $\operatorname{Sim}$ & Não \\
\hline É realizado plane jamento de curto prazo? & $\mathrm{X}$ & & $\mathrm{X}$ & & $\mathrm{X}$ & & $\mathrm{X}$ & & $\mathrm{X}$ & \\
\hline Os serviços semanais são planejados formalmente? & $\mathrm{X}$ & & & $\mathrm{X}$ & $\mathrm{X}$ & & $\mathrm{X}$ & & $\mathrm{X}$ & \\
\hline Há envolvimento dos encarregados e mestre nesse planejamento? & $\mathrm{X}$ & & $\mathrm{X}$ & & $\mathrm{X}$ & & & $\mathrm{X}$ & $\mathrm{X}$ & \\
\hline Há re união conjunta entre empreiteira e terceirizadas? & & $\mathrm{X}$ & $\mathrm{X}$ & & $\mathrm{X}$ & & $\mathrm{X}$ & & & $\mathrm{X}$ \\
\hline São levantadas possíveis restrições nos planejamentos? & & $\mathrm{X}$ & $\mathrm{X}$ & & & $\mathrm{X}$ & $\mathrm{X}$ & & & $\mathrm{X}$ \\
\hline Existe preocupação em observar as condições de campo para os seviços futuros? & $\mathrm{X}$ & & $\mathrm{X}$ & & $\mathrm{X}$ & & $\mathrm{X}$ & & $\mathrm{X}$ & \\
\hline Os serviços costumam ocorrer no prazo previsto? & & $\mathrm{X}$ & $\mathrm{X}$ & & & $\mathrm{X}$ & & $\mathrm{X}$ & $\mathrm{X}$ & \\
\hline Quando ocorre atraso exis te há investigação do motivo? & $\mathrm{X}$ & & $\mathrm{X}$ & & $\mathrm{X}$ & & $\mathrm{X}$ & & $\mathrm{X}$ & \\
\hline \multirow{2}{*}{ ENCARREGADO OU MESTRE } & \multicolumn{2}{|c|}{ Obra A } & \multicolumn{2}{|c|}{ Obra B } & \multicolumn{2}{|c|}{ Obra C } & \multicolumn{2}{|c|}{ Obra D } & \multicolumn{2}{|c|}{ Obra E } \\
\hline & Sim & Não & $\operatorname{Sim}$ & Não & $\operatorname{Sim}$ & Não & $\operatorname{Sim}$ & Não & $\operatorname{Sim}$ & Não \\
\hline As equipes iniciais são as que fazem o retrabalho? & & $\mathrm{X}$ & $\mathrm{X}$ & & $\mathrm{X}$ & & $\mathrm{X}$ & & $\mathrm{X}$ & \\
\hline Geralmente o operário realiza serviços parecidos? & $\mathrm{X}$ & & $\mathrm{X}$ & & $\mathrm{X}$ & & $\mathrm{X}$ & & $\mathrm{X}$ & \\
\hline O serviço não foi interrompido por modificação de projeto? & $\mathrm{X}$ & & & $\mathrm{X}$ & $\mathrm{X}$ & & & $\mathrm{X}$ & $\mathrm{X}$ & \\
\hline O serviço não foi interrompido por mudança de planos? & & $\mathrm{X}$ & & $\mathrm{X}$ & $\mathrm{X}$ & & $\mathrm{X}$ & & $\mathrm{X}$ & \\
\hline Nenhum serviço foi interrompido por cruzamento de fluxo com outra equipe? & $\mathrm{X}$ & & & $\mathrm{X}$ & $\mathrm{X}$ & & $\mathrm{X}$ & & $\mathrm{X}$ & \\
\hline Existe avaliação da qualidade dos serviços? & $\mathrm{X}$ & & $\mathrm{X}$ & & $\mathrm{X}$ & & $\mathrm{X}$ & & $\mathrm{X}$ & \\
\hline Existe FVS para os serviços executados? & $\mathrm{X}$ & & $\mathrm{X}$ & & $\mathrm{X}$ & & $\mathrm{X}$ & & $\mathrm{X}$ & \\
\hline Os motivos das não conclus ões são registrados? & $\mathrm{X}$ & & $\mathrm{X}$ & & $\mathrm{X}$ & & $\mathrm{X}$ & & $\mathrm{X}$ & \\
\hline As FVS's são analisadas para os novos plane jamentos? & $\mathrm{X}$ & & $\mathrm{X}$ & & $\mathrm{X}$ & & & $\mathrm{X}$ & $\mathrm{X}$ & \\
\hline Existem ações corretivas com base nas restrições identificadas? & $\mathrm{X}$ & & $\mathrm{X}$ & & $\mathrm{X}$ & & $\mathrm{X}$ & & $\mathrm{X}$ & \\
\hline A qualidade do serviço é inspecionada durante a execução? & & $\mathrm{X}$ & $\mathrm{X}$ & & $\mathrm{X}$ & & & $\mathrm{X}$ & $\mathrm{X}$ & \\
\hline As frentes de trabalho só são liberadas com a aprovação da qualidade das anteriores? & & $\mathrm{X}$ & $\mathrm{X}$ & & $\mathrm{X}$ & & $\mathrm{X}$ & & $\mathrm{X}$ & \\
\hline
\end{tabular}

Figura 12 - Arremate das caixinhas interrompido por falta de placas cerâmicas

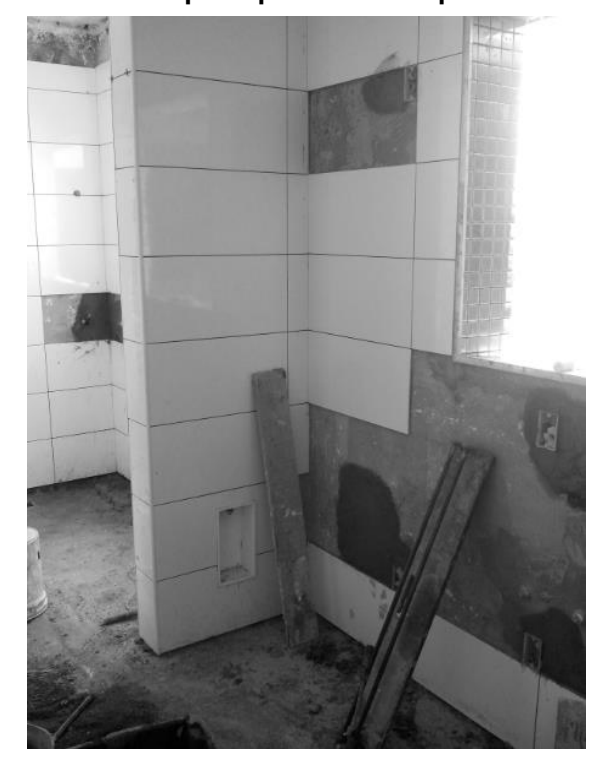

Figura 13 - Categoria de trabalho inacabado nas obras

\begin{tabular}{|c|c|c|c|c|c|c|}
\hline CATEGORIA & Obra A & Obra B & Obra C & Obra D & Obra E & Total \\
\hline Acesso/circulação & & & & & & 0 \\
\hline Ajuste de componentes & $\mathrm{X}$ & & $\mathrm{X}$ & & $\mathrm{X}$ & 3 \\
\hline Área de trabalho & & & & & & 0 \\
\hline Estoque de materiais ou componentes & & $\mathrm{X}$ & & & & 1 \\
\hline Equipamentos/ferramentas & & & & & & 0 \\
\hline Suprimento de água e eletricidade & & & & & & 0 \\
\hline Proteção & & & & & & 0 \\
\hline Sequenciamento & $\mathrm{X}$ & & & $\mathrm{X}$ & & 2 \\
\hline
\end{tabular}

48 Santos, P. R. R.; Santos, D. de G. 
Figura 14 - Pré-requisitos ligados ao trabalho inacabado nas obras

\begin{tabular}{|c|c|c|c|c|c|c|}
\hline PRÉ-REQUISITOS & Obra A & Obra B & Obra C & Obra D & Obra E & Total \\
\hline Informação & & $\mathrm{X}$ & & & & 1 \\
\hline Materiais e componentes & $\mathrm{X}$ & $\mathrm{X}$ & & & & 2 \\
\hline Mão de obra & $\mathrm{X}$ & & $\mathrm{X}$ & & $\mathrm{X}$ & 3 \\
\hline Equipamentos/ferramentas & & & & & & 0 \\
\hline Espaço & & & & & & 0 \\
\hline Serviços interligados & $\mathrm{X}$ & & $\mathrm{X}$ & $\mathrm{X}$ & $\mathrm{X}$ & 4 \\
\hline Condições externas & & & & & & 0 \\
\hline Infraestrutura do espaço de trabalho & & & & & & 0 \\
\hline
\end{tabular}

A categoria "ajuste de componentes" foi atribuída a três casos e estava intimamente ligada com a qualidade dos produtos anteriores e a incidência de making-do. Pode-se ligá-la também aos prérequisitos "materiais e componentes" e à "mão de obra", que ao apresentarem falha de qualidade prejudicaram a interligação entre os serviços - prérequisito dos "serviços interligados". O trabalho inacabado por ocorrência de falta de sequenciamento aconteceu em duas obras, estando também relacionado à deficiência na ligação entre serviços.

A categoria estoque de materiais foi encontrada em somente uma obra (obra B), resultado de falha logística no gerenciamento de insumos. Essa mesma falha fez com que faltasse o pré-requisito "informação" para a obra em questão. No caso, a execução do revestimento de paredes teve que ser interrompida por alterações tardias de projetos de instalações, durante o curso da obra.

Os impactos notados encontram-se na Figura 15. A falta de terminalidade foi notada nas cinco obras, seguida da diminuição da produtividade $\mathrm{e}$ desmotivação, encontradas em quatro dos estudos. Esses dois últimos fatores incidem em conjunto, ou seja, a queda de produtividade proveniente dos serviços incompletos, seja por falta de terminalidade ou por retrabalho, desmotivou as equipes envolvidas, que visavam a remuneração por produção.

O retrabalho fez-se presente na maioria das obras e foi sempre associado às perdas de materiais. Ele também foi consequência do making-do, nas duas obras que esse tipo de perda ocorreu, acompanhado da redução da qualidade do serviço.

O impacto no tempo de ciclo dos pacotes informais foi resumido na Figura 16. Os fatores relacionados a esse aumento do tempo de ciclo foram a diminuição da produtividade, principalmente, e o retrabalho.

Há de se frisar também que o PCP influiu diretamente na proporção encontrada, já que o cálculo foi feito a partir do tempo de ciclo programado nas reuniões. Assim percebe-se que a obra B e a obra E tiveram os menores impactos pelo alto nível de comprometimento e precisão dos planos de curto prazo. A obra $\mathrm{B}$, inclusive, não apresentou acréscimo de tempo com relação ao programado. Outro fato em comum nas duas obras foi o tempo de ciclo curto, fator que facilita a gestão das restrições.

\section{Conclusões}

A investigação de perdas por trabalho inacabado ocorreu satisfatoriamente, tendo em vista que vários exemplos foram encontrados nas obras pesquisadas. A metodologia aplicada nas visitas ajudou na identificação dos motivos para a existência do trabalho inacabado e na influência do PCP para a sua ocorrência. A análise do impacto no tempo de ciclo conseguiu aferir as parcelas de descontinuidades do trabalho observadas em campo, em detrimento do prazo estimado pelos engenheiros.

Os exemplos de trabalho inacabado observados se assemelharam com os exemplos citados na literatura (FIREMAN, 2012), sendo em sua maioria ligados à má execução das tarefas anteriores. Os pacotes resultantes de falta de terminalidade foram vistos em todas as obras, revelando que as falhas no sequenciamento das tarefas associadas com falhas na gestão da qualidade serviram de desencadeadores para o trabalho inacabado.

A associação do trabalho inacabado com o making-do, quando observada, foi fruto da improvisação da mão de obra para contornar a baixa qualidade dos produtos/processos anteriores (SOMMER, 2010), ligando-se aos ajustes dos componentes construtivos para a conclusão das tarefas. A mão de obra também contribuiu nos casos de pacotes informais de retrabalho, devido à má qualidade do serviço. Entretanto, esse problema foi também ligado à gestão da qualidade. 
Figura 15 - Impactos do trabalho inacabado nas obras

\begin{tabular}{|c|c|c|c|c|c|c|}
\hline IMPACTOS & Obra A & Obra B & Obra C & Obra D & Obra E & Total \\
\hline Diminuição da produtividade & $\mathrm{X}$ & & $\mathrm{X}$ & $\mathrm{X}$ & $\mathrm{X}$ & 4 \\
\hline Redução da qualidade & $\mathrm{X}$ & & $\mathrm{X}$ & & & 2 \\
\hline Retrabalho & $\mathrm{X}$ & & $\mathrm{X}$ & & $\mathrm{X}$ & 3 \\
\hline Perda de material & $\mathrm{X}$ & & $\mathrm{X}$ & & $\mathrm{X}$ & 3 \\
\hline Redução de segurança & & & & & & 0 \\
\hline Desmotivação & $\mathrm{X}$ & $\mathrm{X}$ & & $\mathrm{X}$ & $\mathrm{X}$ & 4 \\
\hline Falta de terminalidade & $\mathrm{X}$ & $\mathrm{X}$ & $\mathrm{X}$ & $\mathrm{X}$ & $\mathrm{X}$ & 5 \\
\hline
\end{tabular}

Figura 16 - Impacto no tempo de ciclo nas obras

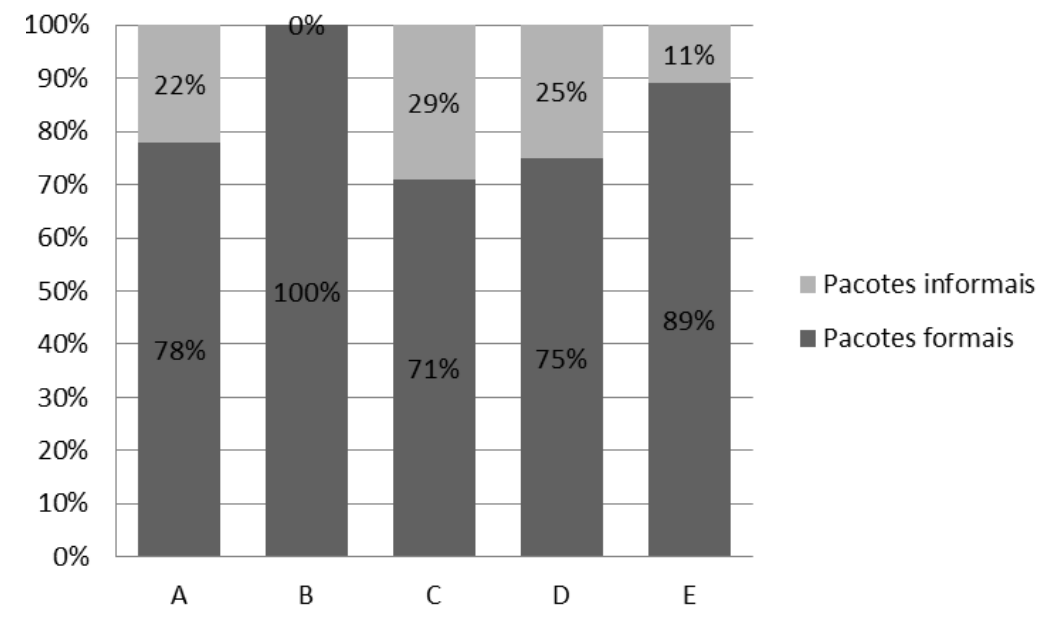

Notou-se que o PCP tem grande influência para o trabalho inacabado. Os canteiros que apresentaram planos de curto prazo formalizados, com participação nas reuniões de funcionários ligados ao nível tático e operacional, atenuaram os impactos desse tipo de perda. Outro fator importante para a redução de perda foi o controle da qualidade rígido, que em alguns casos conseguiu eliminar os pacotes informais devidos ao retrabalho.

Os impactos decorrentes do trabalho inacabado afetaram, além da terminalidade do produto, a mão de obra, que alegou desmotivação por não conseguir completar o trabalho ou por ter que refazê-lo. Junto com a desmotivação veio também a queda da produtividade e a queda na qualidade. Por sua vez, essa redução da qualidade implicou retrabalhos, o que significou, além de perdas de mão de obra, perdas de materiais.

Uma medida geral dos impactos citados foi a análise do tempo de ciclo. Na maioria das obras o tempo de ciclo previsto pelo planejamento de curto prazo não foi cumprido. Em resumo, as obras que falharam no controle da produção e na gestão da qualidade tiveram um acréscimo maior no tempo de execução do serviço. Vale destacar também a relação entre o número de funcionários ligados à gestão da obra e a influência no tempo de ciclo. As obras que tinham equipe administrativa mais encorpada obtiveram impactos menores.

\section{Referências}

ALVES, T. C. L. Diretrizes Para a Gestão dos Fluxos Físicos em Canteiros de Obras: proposta baseada em estudos de caso. Porto Alegre, 2000. 152 f. Dissertação (Mestrado em Engenharia Civil) - Programa de Pós-Graduação em Engenharia Civil, Universidade Federal do Rio Grande do Sul, Porto Alegre, 2000.

BARBOSA, G. et al. Implementing Lean Construction effectively in a year in a construction Project. In: ANNUAL CONFERENCE OF THE INTERNATIONAL GROUP FOR LEAN CONSTRUCTION, $21^{\text {th }}$, Fortaleza, 2013. Proceedings... Fortaleza, 2013.

CUNHA, M. P. Bricolage in Organizations. Instituto Nova Fórum. Lisboa: Universidade Nova de Lisboa, 2004. 
FIREMAN, M. C. T. Proposta de Método de Controle Integrado da Produção e Qualidade, Com Ênfase na Medição de Perdas Por MakingDo e Retrabalho. Porto Alegre, 2012. Dissertação (Mestrado em Engenharia Civil) - Programa de Pós-Graduação em Engenharia Civil, Universidade Federal do Rio Grande do Sul, Porto Alegre, 2012.

FIREMAN, M. C. T. et al. Integrating Production and Quality Control: monitoring making-do and unfinished work. In: ANNUAL CONFERENCE OF THE INTERNATIONAL GROUP FOR LEAN CONSTRUCTION, $21^{\text {th }}$, Fortaleza, 2013. Proceedings... Fortaleza, 2013.

FORMOSO, C. T. et al. An Exploratory Study on the Measurement and Analysis of Making-Do in Construction Sites. In: ANNUAL CONFERENCE OF THE INTERNATIONAL GROUP FOR LEAN CONSTRUCTION, 19 ${ }^{\text {th }}$, Lima, 2011. Proceedings... Lima, 2011.

KOSKELA, L. An Exploration Towards a Production Theory and Its Application to Construction. Espoo, 2000. Thesis (Ph.D) Technical Research Centre of Finland, Espoo, 2000.

KOSKELA, L. Application of the New Production Philosophy to Construction. Technical Report $n^{\circ}$. 72. Center for Integrated Facility Engineering. Stanford University, 1992.

KOSKELA, L. Making-Do: the eighth category of waste. In: ANNUAL CONFERENCE ON THE INTERNATIONAL GROUP FOR LEAN CONSTRUCTION, 12 $2^{\text {th }}$, Elsinore, 2004. Proceedings... Denmark, 2004.

LEÃO, C. F. Proposta de Modelo Para Controle Integrado da Produção e da Qualidade Utilizando Tecnologia de Informação. Porto Alegre, 2014. 179 f. Dissertação (Mestrado em Engenharia) - Programa de Pós-Graduação em Engenharia Civil, Universidade Federal do Rio Grande do Sul, Porto Alegre, 2014.
MACHADO, R. L. A Sistematização de Antecipações Gerenciais no Planejamento da Produção de Sistemas da Construção Civil. Florianópolis, 2003. Tese (Doutorado em Engenharia de Produção) - Programa de PósGraduação em Engenharia de Produção, Universidade Federal de Santa Catarina, Florianópolis, 2003.

RONEN, B. The Complete kit Concept. The International Journal of Production Research, v. 30, n. 10, p. 2457-2466, 1992.

SANTOS, D. de G. Modelo de Gestão de Processos na Construção Civil Para Identificação de Atividades Facilitadoras. Florianópolis, 2004, 219 f. Tese (Doutorado em Engenharia de Produção) - Programa de PósGraduação em Engenharia de Produção, Universidade Federal de Santa Catarina, Florianópolis, 2004.

SOMMER, L. Contribuições Para Um Método de Identificação de Perdas Por Improvisação em Canteiros de Obras. Porto Alegre, 2010. 150 f. Dissertação (Mestrado em Engenharia Civil) Programa de Pós-Graduação em Engenharia Civil, Universidade Federal do Rio Grande do Sul, Porto Alegre, 2010.

TREVILLE, S.; ANTONAKIS, J. Could Lean Production Job Design be Intrinsically Motivating? Contextual, configurational, and levels-of-analysis issues. Journal of Operations Management, v. 24, n. 2, p. 99-123, 2006.

VIANA, D. D.; FORMOSO, C. F.; KALSAAS, B. T. Waste in Construction: a systematic literature review on empirical studies. In: ANNUAL CONFERENCE OF THE INTERNATIONAL GROUP FOR LEAN CONSTRUCTION, $20^{\text {th }}$, San Diego, 2012. Proceedings... San Diego: State University of San Diego, 2012.

WEICK, K. E. Improvisation as a Mindset for Organizational Analysis. Organization Science, v. 9, n. 6, p. 543-555, 1998. 
Paulo Ricardo Ramos Santos

Departamento de Engenharia Civil, Centro de Ciências Exatas e Tecnologia | Universidade Federal de Sergipe | Av. Marechal Rondon, s/n, Cidade Universitária, Jardim Rosa Elze | Sáo Cristovão - SE - Brasil | CEP 49100-000 | Tel.: (79) 3194-6700 Ramal 6704 |

E-mail: paulo_ricardo.rs@hotmail.com

\section{Débora de Gois Santos}

Departamento de Engenharia Civil, Centro de Ciências Exatas e Tecnologia | Universidade Federal de Sergipe |

E-mail: deboragois@yahoo.com.br

\section{Revista Ambiente Construído}

Associação Nacional de Tecnologia do Ambiente Construído

Av. Osvaldo Aranha, $99-3^{\circ}$ andar, Centro

Porto Alegre - RS - Brasil

CEP 90035-190

Telefone: +55 (51) 3308-4084

Fax: +55 (51) 3308-4054

www.seer.ufrgs.br/ambienteconstruido

E-mail: ambienteconstruido@ufrgs.br

52 Santos, P. R. R.; Santos, D. de G. 